\title{
The Influence of Grain Size on the Toughness of Monolithic Ceramics
}

Experiments have shown that there may be an optimal grain size which maximizes the toughness of polycrystalline ceramics. In this paper, we attempt to develop a theoretical model which can predict the effect of grain size on the toughness of ceramics. We assume that three principal mechanisms affect the toughness of the material: distributed microcracking; crack trapping by tough grains; and frictional energy dissipation as grains are pulled out in the wake of the crack. The grain size influences these mechanisms in several ways. The energy dissipated due to frictional crack bridging increases with the size of the bridging grains, tending to improve toughness. However, as the grain size increases, the density of microcracks in the solid also increases, which eventually weakens the material. In addition, the level of inter-granular residual stress is also reduced by microcracking, which as a detrimental effect on the toughening due to bridging. We have developed a simple model to quantify these effects. However, the model does not predict the dramatic loss of strength which has been observed to occur beyond a critical grain size. We have therefore proposed an alternative explanation for the apparent decrease in toughness in coarse grained ceramics. Calculations indicate that in a coarse grained material, the main contribution to toughness is due to frictional crack bridging. However, to produce this toughening, the bridging zone must be over 500 grains long. In practice, the length of the bridging zone in a coarse grained solid may be comparable to the dimensions of the specimen used to measure its toughness. Under these conditions, it is not appropriate to use the concept of a geometry independent toughness to characterize the strength of the specimen. We have therefore developed a simple model of a double cantilever beam fracture specimen, which accounts for the effects of large scale bridging. Using this model, we are able to predict the apparent decrease in toughness measured in coarse grained specimens.

\section{Introduction}

The strength of ceramic components is almost invariably limited by brittle failure. There is therefore considerable interest in finding methods of improving the fracture properties of ceramics, by designing their microstructure appropriately. While there are many microstructural features which may affect toughness, the grain size of the solid is perhaps the simplest to vary through processing. The objective of this paper is to estimate the optimum grain size for a ceramic component, by modelling the fracture processes theoretically.

Our study is motivated by some experimental measurements reported by Rice et al. (1981), which show that there is a particular grain size with maximizes the strength of ceramic specimens. The experiments involved measuring the fracture toughness of several ceramics as a function of grain size, using double cantilever beam specimens. The results of the experiment are summarized in Fig. 1. It is evident that variations in grain size can produce large changes in toughness, and that

Contributed by the Materials Division and presented at the Symposium on Micromechanics of Ceramics and Ceramic Composites; Winter Annual Meeting, Anaheim, CA, November 8-13, 1992, of The American Society of MechanicaI ENGINEERS. Manuscript received by the Materials Division July 11, 1992; revised manuscript received September 28, 1992. Associate Technical Editor: S. Suresh. there is a particular grain size for which toughness is a maximum. Similar trends have been reported by a number of other investigations (Cook et al., 1985; Swain, 1986; Vekinis et al., 1990), although among these only Rice et al. (1981) have investigated the large range of grain size shown in Fig. 1.

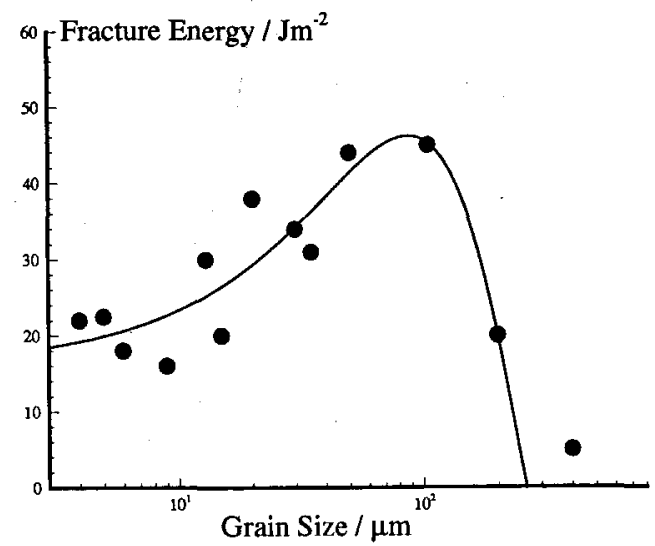

Fig. 1 Measured variation of toughness with grain size for alumina, after Rice et al. (1981) 


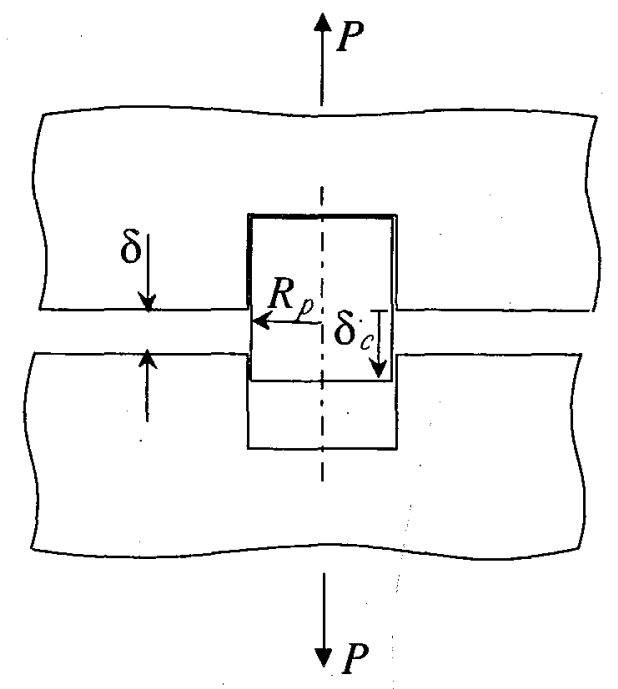

Fig. 2 Model of a grain puiled out in the wake of a crack

Ceramics are sometimes thought of as ideally brittle materials, and at first sight it may appear that their toughness should be independent of grain size. However, a closer examination reveals that, in addition to the work required to create new surfaces, several other mechanisms may dissipate energy as a crack propagates through the solid. Three mechanisms are thought to be particularly important in determining toughness: (i) distributed microcracking near the crack tip; (ii) crack deflection along weak grain boundaries; and (iii) crack trapping combined with crack face bridging by tough grains.

Microcracks near the crack tip have been shown to have two effects. They shield the tip of the crack from remote stress, and so increase toughness (Evans and Faber, 1981; Evans, 1984; Evans and Fu, 1985; Hutchinson, 1987; Ortiz, 1987). However, they may also reduce toughness, since a crack may propagate through the solid by coalescing with successive microcracks ahead of its tip. This process requires less energy than to fracture a homogeneous solid (Ortiz, 1988). In contrast, crack deflection only enhances the effective toughness of the solid. Since the fracture of ceramics is predominantly intergranular, cracks tend to follow a tortuous path through the material. This increases the area of surface to be fractured, and so raises toughness. However, there is evidence to suggest that crack trapping and bridging may be the most effective toughening mechanisms in polycrystalline ceramics. Swanson et al. (1987) and Vekinis et al. (1990) have observed bridging particles in the wake of cracks propagating through alumina. Vekinis and co-workers also measured a substantial increase in toughness as the bridging zone developed. The mechanism responsible for forming bridging particles is not fully understood, but they may be caused by variations in fracture toughness between neighboring grains. In this event, the tough grains behave as tough they were the reinforcing phase in a composite material, and contribute to toughness in two ways. They trap the front of a crack propagating through the solid, so that the crack can only bypass the obstacles if the load is increased (Lange, 1970). In addition, the tough grains remain as bridging particles in the wake of the crack. The bridging grains are progressively pulled free from the matrix as the crack grows, dissipating energy by frictional sliding (Vekinis et al., 1990).

The grain size of the solid has only a small influence on the toughening due to crack deflection, but has a strong effect on microcracking and crack bridging. The microcracks in polycrystalline ceramics are believed to be a consequence of microstructural residual stresses in the solid. Tensile stresses acting on the boundaries between adjacent grains can cause spontaneous cracking along the grain facets (e.g., Kuszyk and Bradt,
1973; Cleveland and Bradt, 1978; Fu and Evans, 1985; Fredrich and Wong, 1986). As the grain size is increased, the grain facets become more likely to fracture. Therefore, at low grain sizes, the microcrack density in the solid is small. In this case, the microcracks do not weaken the solid and may even produce a small increase in toughness due to shielding. However, in a specimen with a large grain size, the microcrack density is sufficiently large for the weakening effect of the cracks to outweigh any beneficial effect of shielding. Indeed, there appears to be a critical grain size where the solid disintegrates spontaneously when it is cooled to room temperature after fabrication (Kuszyk and Bradt, 1973; Rice and Pohanka, 1979).

The grain size has two effects on crack bridging, which are best illustrated using the simple model of frictional grain pullout shown in Fig. 2. We idealize the grains by circular cylinders with radius $R_{p}$, and assume they are pinched into the matrix by a compressive thermal residual stress $\sigma_{r}$. We suppose that a portion $\beta$ of the surface of the grain is in contact with the matrix, and is subjected to a frictional traction $f \sigma$, where $f$ is the coefficient of friction. Then, the force required to pull the grain free by a distance $\delta$ is given by

$$
P=P_{0}\left(1-\frac{\delta}{\delta_{c}}\right)
$$

where $\delta_{c}$ is the crack opening displacement required to separate completely the grain from the matrix, and $P_{0}=2 \pi R_{p} \delta_{c} \beta f \sigma_{r}$ is the maximum pinning force on the grain. The effective coefficient of friction $\beta f$ is taken to be an adjustable parameter in our model, to be determined by experiment. The energy dissipated per unit area of crack wake due to frictional grain pullout is

$$
\mathrm{S}^{\text {bridge }}=P_{0} \delta_{c} / 2 L^{2}
$$

where $L$ is the average spacing between bridging grains. Thus, if $\sigma_{r}, \delta_{c} / L$ and $R_{p} / L$ were independent of grain size, the toughening due to crack bridging would increase linearly with grain size. However, the residual stress $\sigma_{r}$ is also influenced by grain size. When the grain size is large, the residual stresses are relaxed by the extensive microcracking in the solid, so that the toughening due to crack bridging is reduced.

In this paper, we describe a model which is used to estimate the toughness of a polycrystalline ceramic. For simplicity, we have neglected the shielding due to microcracking, and the effects of crack deflection: calculations suggest that the toughening due to these mechanisms is small compared to the strengthening due to trapping and bridging (Faber and Evans, 1983; Suresh, 1985; Ortiz, 1987; Bower and Ortiz, 1991). However, the weakening due to microcracking, and the influence of crack trapping and frictional crack bridging are included in our model. The calculation involves three stages. We begin by summarizing a self-consistent model which predicts the microcrack density and residual stress distribution in a polycrystalline ceramic as a function of its grain size. Subsequently, the competing effects of microcracking and crack trapping are investigated, using a three-dimensional model. Finally, the effects of frictional grain bridging are added using a simple cohesive zone approximation, based on the idealized model of frictional grain pull-out illustrated in Fig. 2. By combining the results of these calculations, we are able to estimate the influence of grain size on the fracture toughness of the solid. Our analysis shows that it is possible to account for the increase in toughness with grain size which occurs in fine-grained specimens. However, with any reasonable choice of parameters, our model is not able to account for the loss of toughness in coarse-grained specimens which are observed experimentally.

We conclude by offering an explanation for the discrepancy between theory and experiment. Although our micromechanical model predicts a substantial increase in toughness in a material with a large grain size, the toughness is almost entirely due to frictional crack bridging. In order to produce this tough- 
ening, a large bridged zone (10 to $20 \mathrm{~mm}$ in length) must form in the wake of the crack. Unless the specimen is very much larger than this, the full extent of toughening due to crack bridging cannot be developed. Furthermore, if the size of the specimen is comparable to the length of the bridging zone, it is questionable to interpret the strength of the specimen in terms of a geometry independent fracture toughness.

We have therefore developed a simple model of the double cantilever beam specimens used by Rice et al. (1981), which takes into account the possibility of large scale crack bridging in coarse grained specimens. Using this model, we have been able to account for the apparent sharp downturn in toughness in coarse grained specimens which they observed experimentally.

\section{Residual Stresses and Microcracking}

The residual stress distribution and microcrack density in a ceramic specimen have a strong influence on its toughness. We begin, therefore, by estimating the density of microcracks and levels of residual stress which may be expected in a polycrystalline ceramic, as a function of its grain size.

There are two possible sources of microcracks in ceramics which are of interest here. The microcracks may be nucleated during fabrication: tensile residual stresses are generated during cooling due to the elastic and thermal anisotropy of the grains, which may cause the grain facets to fracture. In addition, further microcracks may be nucleated near the tip of a large crack in the specimen, due to the high levels of stress near the crack tip. The additional microcracks near the crack tip tend to shield it from remote stress, and may cause some toughening. However, calculations suggest that the effect of shielding is small when compared to other mechanisms of toughening such as frictional crack bridging, or crack trapping. For simplicity, we will therefore neglect the additional microcracks which may form near the tip of a crack, and assume that all the microcracks in the solid are nucleated spontaneously during fabrication.

Several authors have pointed out that the extent of spontaneous microcracking in a polycrystalline ceramic is strongly dependent on its grain size (Kuzzyk and Bradt, 1973; Pohanka et al., 1976; Siebenek et al., 1976; Cleveland and Bradt, 1978). A first-order estimate of the extent of thermally induced microcracking can be readily derived as follows. The first step is to calculate the magnitude of the thermal residual stresses in the absence of any microcracking. To this end, we idealize a polycrystalline ceramic as a two-dimensional assembly of hexagonal grains. For simplicity, we assume that all the grains are the same size, which may be characterized by the length of a grain facet $a$. The grains are thermally and elastically anisotropic, and the directions of principal axes of anisotropy vary randomly from one grain to the next. Ortiz and Suresh (1990) have presented detailed numerical simulations of the intergranular residual stresses which develop in this system when the grains are cooled from their fabrication temperature. Their results suggest that the probability $\rho(\sigma)$ of a finding a given level of residual stress $\sigma$ at a particular point in the solid is closely approximated by a normal distribution. For the present purposes, we wish to calculate the probability $\rho\left(\sigma_{n}\right)$ of a finding a given level of stress $\sigma_{n}$ acting perpendicular to a grain facet. Provided that the distribution of grain orientations is isotropic, it follows that $\mathcal{P}\left(\sigma_{n}\right)$ is also normal, and may be expressed as

$$
\mathcal{P}\left(\sigma_{n}\right)=\frac{1}{\sqrt{2 \pi \hat{\sigma}}} \exp \left(-\frac{\sigma_{n}^{2}}{2 \hat{\sigma}^{2}}\right)
$$

where $\hat{\sigma}$ denotes the covariance of the distribution. Since $\mathcal{P}\left(\sigma_{n}\right)$ must be independent of the orientation of the grain boundaries, $\hat{\sigma}$ is equivalent to the covariance of any one component of stress in the solid.
The covariance of the stress distribution $\hat{\sigma}$ thus provides a scalar measure of the magnitude of the residual stresses in the solid. Using a self-consistent method, Ortiz and Molinari (1988) have derived expressions for $\hat{\sigma}$ for the case of a thermally anisotropic but elastically isotropic solid. Their result is

$$
\hat{\sigma}^{2}=\mu^{2} \alpha_{e}^{2} \theta^{2} \frac{32}{225} \frac{\nu^{2}-\nu+1}{(1-\nu)^{2}}
$$

where $\mu$ and $\nu$ are the shear modulus and Poisson's ratio of the solid, $\theta$ is the change in temperature during cooling, and $\alpha_{e}$ is an effective thermal expansion coefficient defined in terms of the principal thermal expansion coefficients $\alpha_{1}, \alpha_{2}$, and $\alpha_{3}$ as

$$
\alpha_{e}^{2}=\frac{2}{3}\left[\left(\alpha_{1}-\alpha_{2}\right)^{2}+\left(\alpha_{2}-\alpha_{3}\right)^{2}+\left(\alpha_{3}-\alpha_{1}\right)^{2}\right]
$$

Boas and Honeycomb (1945) argued, and Ortiz and Molinari (1988) later showed, that in most practical cases it is reasonable to neglect the effect of elastic anisotropy on the residual stress distribution. However, the numerical calculations of Ortiz and Suresh (1990) suggest that for properties typical of structural ceramics, the elastic anisotropy of the grains can significantly increase the magnitude of the residual stresses. In Appendix 1 , we have therefore derived a self-consistent estimate for $\hat{\sigma}$ which accounts for both thermal and elastic anisotropy. The main result is that (2.2) remains valid provided that $\mu$ and $\nu$ are interpreted as the effective moduli of the polycrystal and $\alpha_{e}$ is computed from an effective thermal expansion tensor, given in Eq. (A7).

In order to predict microcrack densities from these results, we assume that the microcracks nucleate within grain boundaries at a critical normal stress $\sigma_{c}$. We further assume that, after nucleation, microcracks immediately extend over a complete grain facet and remain stable thereafter. Past work has shown that there is a strong dependence of $\sigma_{c}$ on the grain size $a$ (Rice and Pohanka, 1979; Singh et al., 1979; Krstic, 1984; Fu and Evans, 1985). A widely accepted conjecture is that $\sigma_{c}$ is inversely proportional to $\sqrt{a}$. For example, if we assume that microcracks nucleate when the elastic energy released during their growth exceeds the surface energy of a grain facet, we find (Ortiz and Molinari, 1988)

$$
\sigma_{c}=\sqrt{\frac{C E \gamma}{\left(1-\nu^{2}\right) a}}, \quad C=\frac{3 \pi \sqrt{n}}{8}
$$

Here $E$ and $\nu$ are the Young's modulus and Poisson's ratio of the ceramic, $\gamma$ is the grain boundary surface energy, and $n$ is the number of facets per grain. Three-dimensional grains are sometimes represented as tetrakaidecahedra (Kingery et al., 1976), which have six square and eight hexagonal faces, giving $n=14$. The inverse relation between $\sigma_{c}$ and $\sqrt{a}$ is evident from (2.4). The energy balance argument leading to (2.4) has often been used to determine a critical grain size for spontaneous cracking (Clarke, 1964; Davidge and Green, 1968; Kuszyk and Bradt, 1973; Cleveland and Bradt, 1978; Evans, 1978).

Equations (2.1-2.4) may now be combined to calculate the fraction $q$ of grain facets which are likely to fracture during cooling. If we assume that the facets break whenever the stress perpendicular to the grain boundary $\sigma_{n}$ exceeds the fracture stress $\sigma_{c}$, the fraction of broken facets is given by the area under the distribution $\mathcal{P}\left(\sigma_{n}\right)$ over the interval $\sigma_{n} \geq \sigma_{c}$. Therefore, we may write

$$
q\left(\hat{\sigma} / \sigma_{\epsilon}\right)=\frac{1}{\sqrt{2 \pi}} \int_{\sigma_{c^{\prime}} / \bar{\sigma}}^{\infty} \exp \left(-x^{2} / 2\right) d x
$$

Evidently, $q$ is an increasing function of the magnitude of the residual stresses $\hat{\sigma}$.

Initially, as the temperature is reduced from its fabrication level, $\hat{\sigma}$ grows in proportion to the temperature drop $\theta$. As the stress increases, so the microcrack density also grows, as pre- 


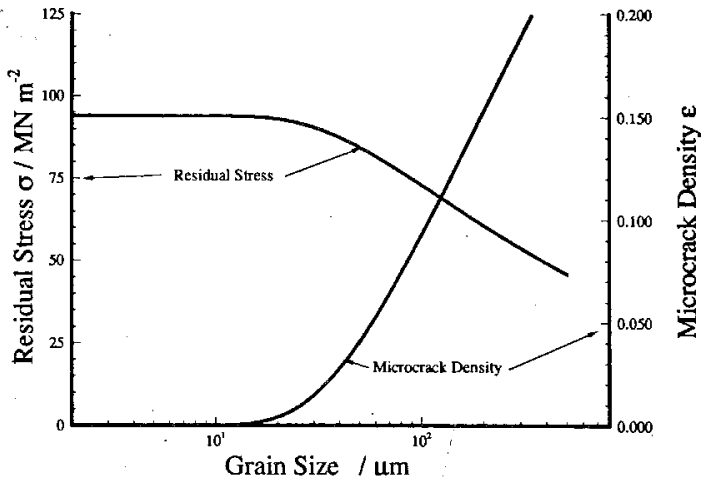

Fig. 3 Predicted variation of microcrack density and residual stress level with grain size for alumina

dicted by (2.5). However, the microcracks tend to relax the residual stresses, so that $\hat{\sigma}$ must be regarded as a function of $q$. An estimate of the extent to which the residual stresses are relaxed due to microcracking can be obtained by using formulae for the effective elastic moduli of a solid containing isotropic distribution of penny-shaped cracks, given by $\mathrm{Bu}$ diansky and O'Connell (1976). These formulae are

$$
\begin{aligned}
& \frac{E}{E_{0}}=1-\frac{16}{45} \frac{\left(1-\nu^{2}\right)(10-3 \nu)}{2-\nu} \epsilon \\
& \frac{\mu}{\mu_{0}}=1-\frac{32}{45} \frac{\left(1-\nu^{2}\right)(5-\nu)}{2-\nu} \epsilon \\
& \epsilon=\frac{45}{16} \frac{\left(\nu_{0}-\nu\right)(2-\nu)}{\left(1-\nu^{2}\right)\left[10 \nu_{0}-\nu\left(1+3 \nu_{0}\right)\right]}
\end{aligned}
$$

Here, $\epsilon=N R_{m}^{3}$ is Budiansky and O'Connell's measure of microcrack density, in which $N$ is the number of microcracks per unit volume, and $R_{m}$ is the radius of the cracks. $E_{0}, \mu_{0}$, and $\nu_{0}$ denote the Young's modulus, shear modulus and Poisson's ratio of the uncracked solid, while $E, \mu$, and $\nu$ are the elastic constants for the microcracked material. Ortiz and Molinari (1988) have estimated the relation between $q$ and $\epsilon$ to be

$$
\epsilon=\frac{3}{\pi \sqrt{n}} q
$$

where $n$ is the number of facets per grain. For $n=14,(2.7)$ becomes $\epsilon \approx 0.255 q$. Within a self-consistent framework, the stress relaxation due microcracking can be accounted for by using Eqs. (2.6) for the elastic moduli in the expression for $\hat{\sigma}$ in (2.2). Clearly, as the elastic moduli are reduced due to microcracking, so the level of residual stress $\hat{\sigma}$ also decreases.

Given the initial elastic constants $E_{0}, \mu_{0}$, and $\nu_{0}$; the surface energy $\gamma$; the thermal expansion coefficient $\alpha_{e}$; the temperature drop during fabrication $\theta$ and the grain size $a$, Eqs. (2.1-2.7) may be solved for the microcrack density $\epsilon$. The level of residual stress $\hat{\sigma}$ and effective elastic constants for the microcracked solid also follow as part of the solution. By way of illustration, we have plotted the variation of microcrack density $\epsilon$ and residual stress $\hat{\sigma}$ as a function of grain size in Fig. 3, using parameters which are representative of polycrystalline alumina. The results show that the microcrack density is small for a fine grained solid, but increases sharply beyond a critical grain size. At the same time, the residual stress begins to decrease. The trends shown in Fig. 3 are in good agreement with more accurate finite element calculations reported by Ortiz and Suresh (1990). The level of residual stress is also comparable to the experimental measurements by Blendell and Coble (1982).

\section{Calculation of Effective Toughness}

The method outlined in the preceding section allows the residual stress distribution and microcrack density in a polycrystalline ceramic to be calculated as a function of its grain size. The next step is to estimate the effective toughness of the solid. We assume that three mechanisms affect the toughness of the solid: tough grains trap the crack front, tending to increasing toughness, while microcracks in the solid provide a weak path for crack growth and so reduce the material's strength. The third contribution to toughness is the energy dissipated as grains are pulled out in the crack wake.

We have used the simple three-dimensional model illustrated in Fig. 4 to account for all three mechanisms. We suppose that the solid contains a semi-infinite crack on the plane $x_{3}=0$. The crack front is initially straight and parallel to the $x_{2}$ axis. The solid is elastically homogeneous and isotropic, and is assumed to be ideally brittle with an intrinsic toughness $\mathcal{G}_{c}^{\text {mat }}$. We will assume that the intrinsic toughness is twice the surface energy of the solid $\mathcal{G}_{c}^{\text {mat }}=2 \gamma$. In addition, the solid contains a doubly periodic distribution of particles with spacing $L$ ahead of the crack front. The particles are intended to model grains whose toughness $\mathcal{G}_{c}^{\text {par }}$ exceeds that of neighboring grains, so that they are left as bridging particles in the wake of the crack. Their volume fraction is regarded as an adjustable parameter, to be determined by experiment. For simplicity, we idealize the tough particles as cylindrical, with the axis of each cylinder parallel to the $x_{3}$ axis. The $x_{3}=0$ plane therefore contains a distribution of circular regions, radius $R_{p}$, where the toughness exceeds that of a matrix. In addition, the solid contains a regular distribution of microcracks, which are idealized as penny shaped cracks, radius $R_{m}$, coplanar with the semi-infinite crack. The microcracks are assumed to be midway between neighboring particles, as illustrated in Fig. 4.

We now imagine that the solid is subjected to remote loading, so that a distribution of mode I stress intensity factors $K(s)$ is induced along the semi-infinite crack. The magnitude of the remote load is parameterized by a remote energy release rate $\mathrm{S}^{\infty}$, defined by

$$
\mathcal{G}^{\infty}=\frac{1}{L} \int_{0}^{L} \mathcal{S}\left(x_{2}\right) d x_{2},
$$

where $\mathcal{S}\left(x_{2}\right)$ denotes the variation of local energy release rate along the semi-infinite crack, and $L$ denotes one wavelength of the crack front. When the remote load reaches a critical magnitude, the semi-infinite crack starts to propagate through the solid. The symmetry of the configuration ensures that the crack is not deflected from its initial plane, but the crack front does not remain straight. Parts of the crack which contact the tough particles arrest, while the remainder of the crack front bows out between the trapping particles. In addition, the microcracks tend to attract the front of the crack, and so weaken the solid. Eventually, the crack front bypasses the tough particles, either by coalescing with itself on the far side of the particles, or else by coalescing with the microcracks. In either event, a row of tough grains is left in the crack wake. In our model, we assume that the grains debond from the matrix soon after they are bypassed, and are progressively pulled free as the crack faces separate. Several rows of bridging particles may be formed in the crack wake in this way. Eventually, a steady state is reached where new bridging grains for at the crack tip at the same rate as they are lost in the crack wake. Our objective is to calculate the variation of remote load (and hence the effective toughness) as the crack propagates through the solid. It is convenient to consider the effects of crack trapping and frictional crack bridging separately.

3.1 The Influence of Crack Trapping and Microcracking on Toughness. The effects of crack trapping can only be investigated by calculating in three dimensions the shape of the crack as it propagates through the solid. An approach to solving problems of this nature has been devised by Rice (1985, 1989), and Gao and Rice (1986, 1987), who found a method 

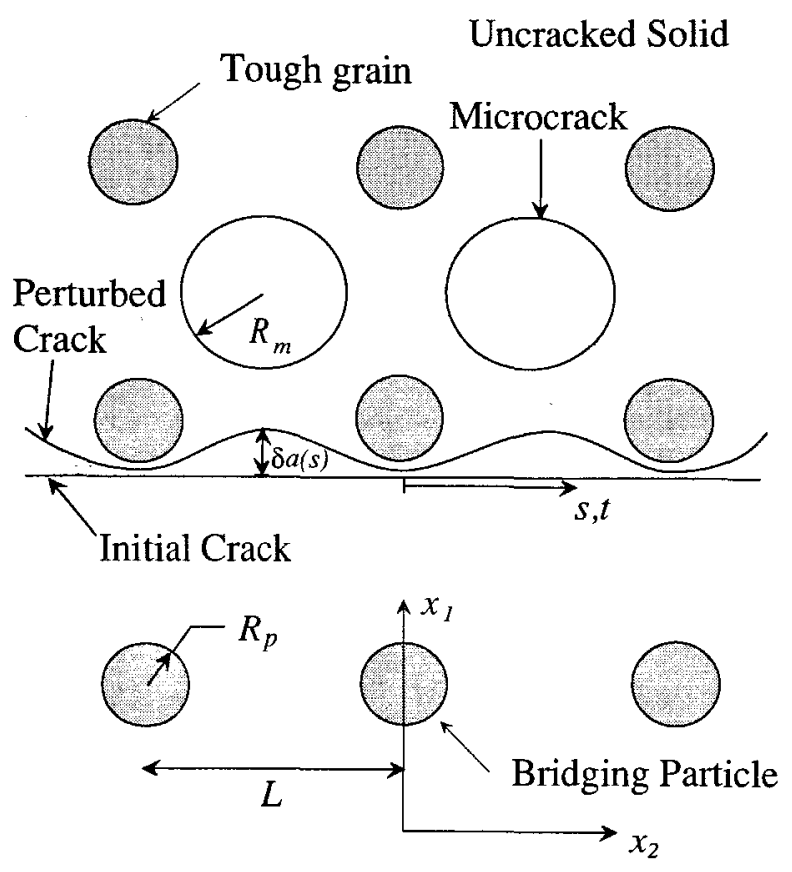

\section{Cracked Solid}

Fig. 4 Idealized model used to estimate the toughening due to crack trapping, bridging and microcracking



Fig. 5 The shape of a semi-infinite crack as it propagates through a solid containing microcracks and tough particles

for calculating the change in stress intensity factor caused by a small change in the geometry of a crack. The method has been extended to arbitrarily large changes in crack shape by Bower and Ortiz (1990). By applying a succession of small perturbations to the initially straight semi-infinite crack, it is possible to calculate the variation of stress intensity factor along the crack front as it bows between the particles. The shape of the semi-infinite crack must be found as part of the solution. The configuration of the crack is determined from the condition that $\mathrm{G}(s)=\mathrm{G}^{\text {mat }}$ on all propagating regions of the crack. Once the distribution of stress intensity factor has been found, the toughening due to trapping may be calculated using Eq. (3.1). To save space, we will not describe the theory involved in the incremental perturbation method here: a full discussion is given in Bower and Ortiz $(1990,1991)$.

The results of our numerical simulations of a semi-infinite crack interacting with tough particles and microcracks are summarized in Figs. 5 to 7 . Figure 5 shows the behavior of the crack as it bypasses two successive rows of tough particles, for a particle radius $R_{p} / L=0.1$ and microcrack radius $R_{m} / L=0.3$. Crack profiles are shown at various magnitudes of remote stress

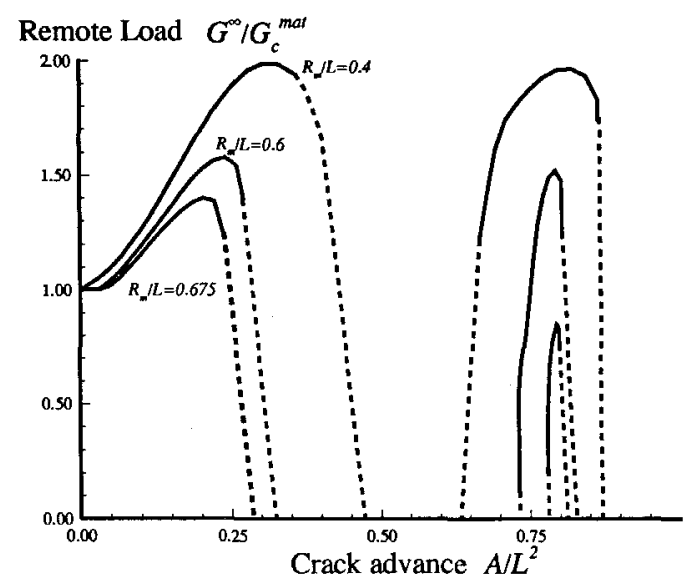

Fig. 6 The variation of remote load as a crack bypasses three rows of tough particles, for various microcrack densities

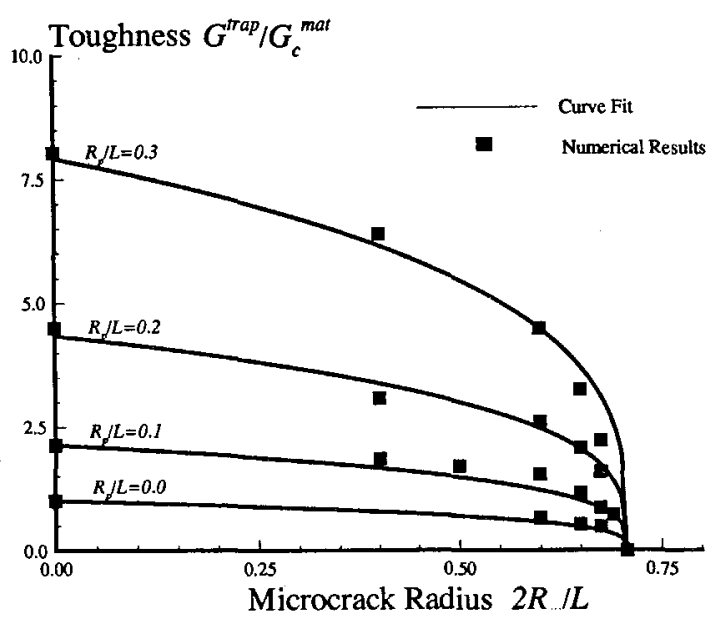

Fig. 7 The variation of toughness due to trapping alone, as a function of microcrack radius

$\mathrm{S}^{\infty} / \mathrm{S}_{c}^{\text {mat }}$. The tough particles trap the front of the crack, causing it to bow out between them as the remote load increases. The crack can bypass the obstacles in one of two ways. If the microcracks are small, the crack eventually coalesces with itself on the far side of the tough particles. Alternatively, if the microcrack radius exceeds a critical value, the crack is attracted by the microcracks and eventually coalesces with them. Our simulations indicate that for a particle radius $R_{p} / L=0.1$, the critical microcrack radius is of the order $R_{m} / L=0.2$. Consequently, in the simulation shown in Fig. 3 the crack eventually advances far enough to be attracted by the row of microcracks. The remote load begins to decrease at this point, and drops to zero when the semi-infinite crack and microcracks coalesce.

The process of coalescence cannot be analyzed in detail using the incremental perturbation method used here, due to numerical difficulties. We therefore resume the analysis at a later stage, as illustrated in Fig. 5. The first row of tough particles is left as a row of bridging grains in the crack wake. For simplicity, we have assumed that the pinning force on the particles $P=0$ in this case, so they do not contribute to toughness or influence the crack shape. The semi-infinite crack is trapped by the second row of particles, but its front is not straight at the instant of contact. Instead, part of the crack front lies along the edge of the first row of microcracks. As the remote load is increased, the crack bows beyond the second row of particles, and eventually coalesces with the second row of microcracks. 
Figure 6 shows the variation of remote load as the crack bypasses three successive rows of particles. For simplicity, the results neglect the effect of pinning particles in the crack wake. The load is plotted as a function of $A / L^{2}$, where $A$ denotes the area swept by one wavelength of the crack front. To examine the influence of microcracking, we have shown results for three microcrack radii. In each case, the particle radius is $R_{p} / L=0.1$. The dashed portion of the curve in Fig. 6 occurs during coalescence, and has not been calculated explicitly. We have made use of the result that $S^{\infty}$ must fall to zero at the point of coalescence to deduce the probable shape of the loadcrack advance curve in this region.

The results in Fig. 6 show clearly the influence of microcracks near the crack tip on the process of crack trapping. While the microcracks are small, the semi-infinite crack bypasses the tough grains by coalescing with itself. Under these conditions, the microcracks do not greatly influence its behavior. The stress intensity factor on the semi-infinite crack is increased slightly by the row of microcracks near its tip. However, this effect is not significant unless the semi-infinite crack is very close to the microcracks. Thus, the maximum load for $R_{m} / L=0$ and $R_{m} / L=0.2$ do not differ greatly. However, if the microcracks are sufficiently large, the crack bypasses the row of particles by coalescing with microcracks in its path. In this case, the peak value of $\mathcal{G}^{\infty}$ is significantly reduced, since the crack only needs to bow for a short distance beyond the particles before it bypasses them. This effect is evident in the results for $R_{m} /$ $L>0.3$ shown in Fig. 6 .

In our model, the maximum value of microcrack radius before neighboring microcracks touch is $R_{m} / L=1 / \sqrt{8}$. As the microcrack radius approaches this value, the crack only needs to advance a short distance to propagate from one row of microcracks to the next. In this case, we find that the peak load required to bypass the first row of particles is greater than the load needed to bypass subsequent rows, as indicated by the results for $R_{m} / L>0.3$ in Fig. 6 . Since the load required to bypass the first row of particles is a consequence of our assumption that the semi-infinite crack is initially straight, we assume that the effective toughness is equal to the maximum load required to bypass subsequent rows of particles.

The influence of crack trapping on toughness is summarized in Fig. 7, which shows the variation of the peak load required to bypass a row of particles, $\mathcal{G}^{\text {trap }} / \mathcal{G}_{c}^{\text {mat }}$ as a function of microcrack radius $R_{m} / L$. Results are shown for various densities of tough grains $R_{p} / L$. The results illustrate clearly the influence of microcracking on the process of crack trapping. For low microcrack densities, the toughness is almost independent of microcrack radius. As the microcrack radius approaches its maximum value $R_{m} / L=1 / \sqrt{8}$, the toughening effect of trapping drops sharply.

To combine the effects of crack trapping with bridging, it is convenient to fit an approximate curve to the results shown in Fig. 7. Some insight into the mechanism of crack trapping is helpful in choosing an appropriate form for the approximation. Bower and Ortiz (1991) give a detailed discussion of the behavior of a crack trapped by rows of tough particles. They show that if the particle toughness $K_{c}^{\text {par }}$ is less than a critical value, the crack penetrates the particles rather than bypassing them. In this case, the toughening can be calculated exactly (Rose, 1975), and is given by

$$
\frac{\mathcal{G}_{c}^{\mathrm{trap}}}{\mathcal{G}_{c}^{\text {mat }}}=\left\{1+\left(\frac{\mathcal{G}_{c}^{\text {par }}}{\mathcal{G}_{c}^{\text {mat }}}-1\right) \frac{2 R_{p}}{L}\right\}
$$

Bower and Ortiz (1991) have calculated the critical value of $S_{c}^{\text {par }}$ for the crack to bypass the tough grains rather than penetrate them. Their results are closely approximated by

$$
G_{c}^{\text {par }} / G_{c}^{\text {mat }} \approx\left(2.1+4.8 R_{p} / L\right)^{2}
$$

If we set $\mathcal{G}_{c}^{\text {par }}$ in (3.2) to be equal to the critical particle tough- ness in (3.3), Eq. (3.2) gives a good approximation to the toughening due to crack trapping in the limit $R_{m} / L=0$. To include the effect of microcracking, we have found that good results are obtained by scaling (3.2) by the distance between neighboring microcracks raised to an appropriate power. Thus, we have approximated the numerical results in Fig. 7 by

$$
\begin{aligned}
\frac{\mathcal{G}_{c}^{\text {trap }}}{\mathcal{G}_{c}^{\text {mat }}}=\left\{1-\frac{2 R_{p}}{L}+\left(2.1+4.8 \frac{R_{p}}{L}\right)^{2} \frac{2 R_{p}}{L}\right\} & \\
& \times\left\{1-2 \sqrt{2} \frac{R_{m}}{L}\right\}^{0.3}
\end{aligned}
$$

The predictions of (3.4) are compared with our numerical results in Fig. 7. It is evident that in the range of $R_{p} / L$ and $R_{m} / L$ considered here, (3.4) is a good approximation to the numerical data.

3.2 Toughening Due to Frictional Crack Bridging. The tough particles which are bypassed by the semi-infinite crack remain intact in its wake, and contribute to toughness by dissipating energy as they are pulled free from the matrix. The toughness progressively increases as successive bridging particles form in the crack wake. A detailed analysis of the resulting $R$-curve behavior has been presented by Bower and Ortiz (1991), who used a three-dimensional model to calculate the variation of toughness with crack length as the bridging zone developed. We will not repeat their analysis here: instead we will derive an expression which gives the steady state toughness once the bridging zone has developed fully.

Both theory and experiment (Vekinis et al., 1990; Bower and Ortiz, 1991) suggest that the length of the bridged zone in the crack wake is several hundred grain diameters in length. Under these conditions, it is not necessary to account for indiyidual bridging particles in the crack wake: their effect may be represented by a distribution of pressure acting on the crack faces. The variation of pressure with crack opening displacement must be chosen to be consistent with the bridging model shown in Fig. 2. By considering the force per unit area of bridged surface, we find that the pressure on the crack faces $p$ varies with crack opening displacement $\delta$ as

$$
p=p_{0}\left(1-\delta / \delta_{c}\right)
$$

where $p_{0}=P_{0} / L^{2}$ is the maximum bridging stress.

If the size of the specimen is large enough to allow the steadystate bridging zone to develop, it is straightforward to combine the effects of trapping and bridging. Imagine that the crack is on the point of bypassing a row of tough grains. Denote the toughening due to trapping, calculated from our numerical results using $\mathrm{Eq}$. (3.1), by $\mathcal{G}_{c}^{\text {trap }}$. Then, using an argument based on evaluating the $J$ integral around a contour which encompasses the crack tip, the flanks of the crack and material distant from the process zone, the effective toughness may be expressed as

$$
\mathcal{S}_{c}^{\mathrm{eff}}=\mathcal{G}_{c}^{\text {trap }}+\mathcal{S}_{c}^{\text {bridge }},
$$

where $g^{\text {bridge }}=p_{0} \delta_{c} / 2$ is the contribution to toughness due to bridging.

3.3 The Variation of Toughness with Grain Size. To find the variation of toughness with grain size, we combine the expression for toughness given in Eq. (3.6), (3.4), and (1.2) with the estimates of microcrack density and residual stress given in Section 2. To this end, we must relate the parameter $R_{m} / L$, which characterizes the microcrack density in (3.6), to the fraction of broken grain facets $q$ given in (2.5). A suitable relationship may be found using a straightforward geometrical argument. We recall that Budiansky and O'Connell (1976) use $\epsilon=N R_{m}^{3}$ to characterize the density of microcracks in the solid, where $N$ is the number of microcracks per unit volume. In addition, assume that $\epsilon$ is related to the number of facets per grain $q$ by Eq. (2.7). For $n=14$, this gives $\epsilon \approx 0.255 q$. To relate 


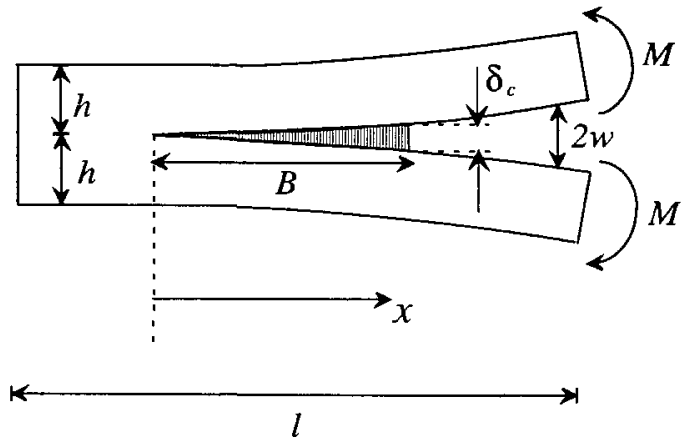

Fig. 8 Idealized model of a double cantilever beam specimen

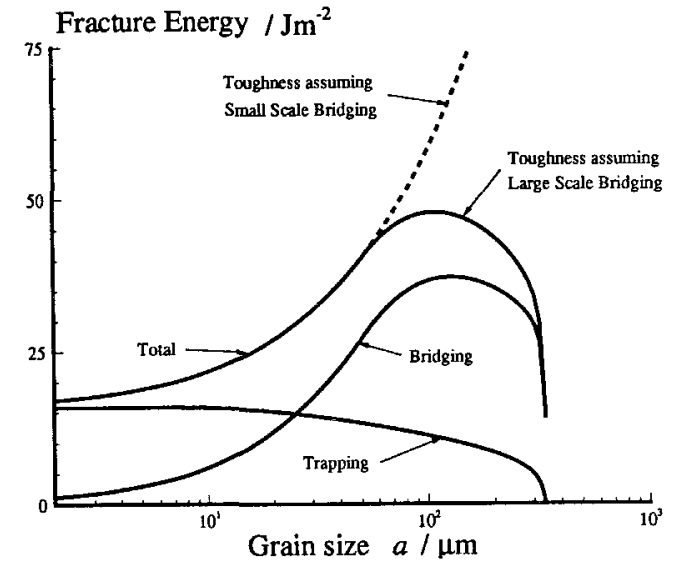

Fig. 9 Predicted variation of toughness with grain size for alumina

$\epsilon$ to $R_{m} / L$, we observe that the total area $\rho$ of the microcracks which intersect unit area of the crack plane is given by Ortiz (1988) as

$$
\rho=\frac{2}{\pi} N R_{m}
$$

In our planar model of crack trapping, $\rho=\pi R_{m}^{2} / L^{2}$. Inserting this relation and the identity $N=\epsilon / R_{m}^{3}$ into (3.7) we find that

$$
\frac{R_{m}}{L}=\sqrt{\frac{2 \epsilon}{\pi}} \approx \sqrt{\frac{q}{2 \pi}} .
$$

In addition, we need to relate the residual stresses $\sigma_{r}$ which pinch the bridging grains into the surrounding matrix to the standard deviation $\hat{\sigma}$ which characterizes the statistical variation of residual stress in the solid. We have taken $\sigma_{r}$ to be the mean value of the compressive stress, so that $\sigma_{r}=0.8 \hat{\sigma}$. Finally, we have set the radius of the pinning particles $R_{p}$ to equal the grain size $a$.

On this basis, we have calculated the variation in toughness with grain size, using parameters in our model intended to be representative of Alumina. The values of most of the parameters may be determined from independent experiments. However, there are two adjustable parameters which can only be determined from fracture toughness measurements: they are the fraction of tough particles $R_{p} / L$ which form bridging grains, and the effective friction coefficient $\beta f$. We have adjusted the value of $R_{p} / L$ to fit the measured toughness for a very fine grained solid. The value of $\beta f$ was then chosen to predict correctly the maximum toughness observed in the experiments.

The values of the parameters are listed in Table 1, and the toughness is plotted as a function of grain size in Fig. 9. For reference, we have also shown the separate contributions to toughness due to trapping $\mathcal{S}^{\text {trap }}$ and bridging $\mathcal{G}^{\text {bridge }}$. For a fine
Table 1 List of parameters used to calculate toughness of

\begin{tabular}{|c|c|}
\hline Parameter & Value \\
\hline Young's modulus, $E_{0}$ & $425 \mathrm{GN} \mathrm{m}^{-2}$ \\
\hline Poisson's ratio $\nu_{0}$ & 0.21 \\
\hline Shear modulus $\mu_{0}=E_{0} / 2(1+\nu)$ & $176 \mathrm{GN} \mathrm{m}^{-2}$ \\
\hline Thermal expansion coefficients: $\alpha_{1}$ & $8.3 \times 10^{-60} \mathrm{C}^{-1}$ \\
\hline$\alpha_{2}$ & $8.3 \times 10^{-60} \mathrm{C}^{-1}$ \\
\hline$\alpha_{3}$ & $9.0 \times 10^{-60} \mathrm{C}^{-1}$ \\
\hline Cool down temperature $\theta$ & $1500^{\circ} \mathrm{C}$ \\
\hline Surface energy $\gamma$ & $1 \mathrm{Jm}^{-2}$ \\
\hline Matrix toughness $\mathcal{S}_{c}^{\mathrm{mat}}=2 \gamma$ & $2 \mathrm{Jm}^{-2}$ \\
\hline No. of facets per grain $n$ & 14 \\
\hline Effective friction coefficient $\beta f$ & 0.045 \\
\hline Fraction of tough grains $R_{p} / L$ & 0.3 \\
\hline Length of cantilever beam specimen $\ell$ & $25 \mathrm{~mm}$ \\
\hline Height of cantilever beam $h$ & $5 \mathrm{~mm}$ \\
\hline Depth of cantilever beam $b$ & $2 \mathrm{~mm}$ \\
\hline
\end{tabular}
Alumina

grained solid, the main contribution to toughness is due to crack trapping. As the grain size increases, the toughness due to trapping decreases due to the increased level of microcracking in the solid. However the decrease in $g^{\text {trap }}$ is outweighed by the increase in frictional energy dissipated in the crack wake. In a coarse grained solid, the main contribution to toughness is frictional crack bridging.

The predictions of our model may be compared with the experimental results of Rice et al. (1981) which are shown in Fig. 1. It is clear that our model predicts correctly the increase in toughness which is observed in fine grained specimens. However, the analysis predicts that the toughness continues to increase beyond a grain size of $100 \mu \mathrm{m}$, whereas the experiments show that the toughness begins to decrease at this point. The values we have assigned to some of the parameters in our model are open to question. However, we have been unable to predict the decrease in toughness observed experimentally with any reasonable choice of the parameters.

It is not difficult to find an explanation for the discrepancy between the measured and predicted toughness. The concept of a specimen geometry independent fracture toughness is only applicable if the length of the bridging zone is small compared to the dimensions of the specimen. Both theory and experiment suggest that the length of the bridging zone required to produce the toughness plotted in Fig. 9 is of the order of 500 grain diameters (Vekinis et al., 1991; Bower and Ortiz, 1991). For a grain size of $100 \mu \mathrm{m}$, this corresponds to a bridged zone over $50 \mathrm{~mm}$ long. The specimens used by Rice et al. (1981) were approximately $25-40 \mathrm{~mm}$ long, so that their results for large grain sizes may not have been obtained under conditions of small scale bridging.

Under large scale bridging conditions, it is not possible to predict the failure load of a component using the standard assumption that fracture energy is independent of specimen geometry. Conversely, the fracture toughness of the material cannot be deduced from the failure load using standard calibrations of stress intensity factors. Therefore, the fracture toughness values quoted by Rice et al. (1981) for coarse grained materials must be interpreted as a measure of the failure load for their particular specimen geometry. In the next section, we attempt to predict this failure load, by developing a simple model which takes into account the geometry of the specimen.

\section{Transition to Large-Scale Bridging}

The specimens used by Rice et al. (1981) in their fracture toughness measurements are illustrated in Fig. 8. Our objective is to calculate the critical moment $M_{f}$ required to fracture the specimens. The value of $M$ at failure may alternatively be expressed as a critical energy release rate $\mathrm{S}_{f}$ using the standard expression for double cantilever beam specimens: 


$$
\mathcal{G}_{f}=M_{f}^{2} / E I b
$$

where $I=b h^{3} / 12$ is the moment of inertia of the beams; $b$ is their width and $h$ their depth, and $E$ is the Young's modulus of the material. While the length of the bridging zone in the crack wake is small compared to the specimen size, $G_{f}$ is equivalent to the effective fracture toughness of the solid $\mathcal{G}_{c}^{\text {eff }}$; otherwise, $\mathcal{G}_{f}<\mathcal{G}_{c}^{\text {eff }}$.

To estimate $\mathcal{G}_{f}$, we assume that the mechanisms of fracture in the specimen are as discussed in the preceding section. Thus, the crack front is trapped by tough grains, and is attracted by microcracks in the solid. In addition, a bridging zone forms in the crack wake. As before, we approximate the bridging particles as a cohesive zone, so that the cohesive stress on the crack faces $p$ is related to the opening displacement $\delta$ by (3.5). We model each arm of the specimen as a beam, so that the equation governing the deflection $w$ of the arms is

$$
\begin{aligned}
& E I w_{, x x x x}+p_{0} b\left(1-\frac{2 w}{\delta_{c}}\right)=0 \quad 0 \leq x \leq B \\
& E I w_{, x x x x}=0 \quad x \geq B
\end{aligned}
$$

Here, $p_{0}=P_{0} / L^{2}$ is the maximum bridging stress, $\delta_{c} \approx a$ is the height of the bridging particles, $B$ is the length of the bridging zone, and the comma denotes differentiation in the usual manner.

The general solution of (4.2) is

$$
w=\frac{\delta_{c}}{2}+A_{1} \cos (\lambda x)+A_{2} \sin (\lambda x)
$$

$$
+A_{3} \cosh (\lambda x)+A_{4} \sinh (\lambda x)
$$

where

$$
\lambda=\left[\frac{2 p_{0} b}{E I \delta_{c}}\right]^{1 / 4}
$$

While the bridging zone is fully contained within the specimen, the constants $A_{1}$ to $A_{4}$ are determined by the following boundary conditions:

$$
\begin{aligned}
& w(0)=0 \\
& w_{, x}(0)=0 \\
& E I w_{, x x}(B)=M \\
& E I w_{, x x x}(B)=0
\end{aligned}
$$

The additional conditions

$$
\begin{aligned}
& \frac{E I}{b} w_{, x x}^{2}(0)=\mathcal{G}_{c}^{\text {trap }} \\
& w(B)=\frac{\delta_{c}}{2}
\end{aligned}
$$

determine the critical moment $M_{f}$ (or $\mathcal{S}_{f}$ ) and the length of the bridging zone $B$. In writing the first of (4.4) we have estimated the bending moment at $x=0$ by equating the energy flux to the crack tip to the toughness due to trapping, using the relationship between $M$ and $\mathcal{G}$ given in (4.1).

The boundary conditions listed in Eq. (4.5) only apply if the bridging zone length $B$ is less than the length of the specimen $\ell$. Otherwise, the tip of the crack reaches the end of the specimen before the grains at $x=\ell$ are fully pulled out of the matrix. In this case, the preceding analysis gives an inadmissible bridging zone length $B>\ell$, and has to be repeated with boundary conditions

$$
\begin{aligned}
& E I w_{, x x}(\ell)=M \\
& E I w_{, x x}(\ell)=0
\end{aligned}
$$

replacing the third and forth of (4.5). A straightforward calculation gives the maximum deflection $w(\ell)$ of the crack faces as

$$
\frac{2 w(\ell)}{\delta_{c}}=\frac{\sin (\lambda \ell) \sinh (\lambda \ell) \sqrt{g}-[1-\cos (\lambda \ell)][1-\cosh (\lambda \ell)]}{\cos (\lambda \ell)+\cosh (\lambda \ell)}
$$

where $g=2 \mathcal{G}_{c}^{\text {trap }} / p_{0} \delta_{c}$. The failure moment $M_{f}$ and hence $\mathcal{S}_{f}$ may then be calculated by a standard application of the $J$ integral, with the result

$$
\mathcal{S}_{f}=\frac{M_{f}}{E I b^{2}}=\mathcal{G}_{c}^{\text {trap }}+\left(2-\frac{2 w(\ell)}{\delta_{c}}\right) \frac{2 w(\ell)}{\delta_{c}} \mathcal{G}_{c}^{\text {bridge }}
$$

where $G_{c}^{\text {bridge }}$ is given in Eq. (1.2). Because of the constraint $2 w(\ell) / \delta_{c} \leq 1$, it is evident from (4.9) that the bridging contribution to $\mathcal{G}_{c}$ is diminished by the transition to large-scale bridging.

We have added the predictions of Eq. (4.9) to Fig. 9, which shows the variation of fracture energy $G_{f}$ with grain size. The dimensions of the specimen used in this calculation were taken from Rice et al. (1981), and are listed in Table 1. Below a grain size of approximately $80 \mu \mathrm{m}$, the steady-state bridging zone is shorter than the length of the specimen, and the full toughness due to bridging is developed. Under these conditions, $G_{f}=G_{c}^{\text {eff }}$. For grain sizes larger than $100 \mu \mathrm{m}$, the bridging zone cannot reach its steady state length, and frictional crack bridging becomes progressively less effective. In addition, the toughening due to crack trapping begins to decrease rapidly, due to the large density of microcracks in the material. These two effects together cause a sharp drop in the apparent toughness of the material $S_{f}$, which is in good agreement with the experimental results.

\section{A P P E N D I X}

\section{Self-Consistent Estimates of the Effect of Elastic Anisotropy on Residual Stresses}

In this Appendix we estimate the effect of elastic anisotropy on thermally induced residual stresses. We consider an infinite solid with elastic moduli $C_{i j k l}$ and thermal expansion coefficients $\alpha_{i j}$, which contains an ellipsoidal domain $\Omega$ with elastic moduli $C_{i j k l}^{*}$ and thermal expansion coefficients $\alpha_{i j}^{*}$. The moduli of the matrix represent the effective properties of the polycrystal, as defined by a self-consistent calculation. Let the body be subjected to a uniform temperature variation $\theta$ and let $\epsilon_{i j}^{0}$ denote a remotely prescribed strain, with a corresponding remote stress $\sigma_{i j}^{0}=C_{i j k l}\left(\epsilon_{k l}^{0}-\theta \alpha_{k l}\right)$. Inside the inhomogeneity $\Omega$, Hooke's law may be written as

$$
\sigma_{i j}^{0}+\Delta \sigma_{i j}=C_{i j k l}^{*}\left(\epsilon_{k l}^{0}-\theta \alpha_{k l}^{*}+\Delta \epsilon_{k l}\right)
$$

where $\Delta \sigma_{i j}$ and $\Delta \epsilon_{k l}$ represent the difference between the remote stress and strain, and the stress and strain inside the inhomogeneity. By introducing a suitable eigenstrain $\Delta \epsilon_{i j}^{*}$ within the inclusion, (A1) may alternatively be written

$$
\sigma_{i j}^{0}+\Delta \sigma_{i j}=C_{i j k l}\left(\epsilon_{k l}^{0}-\theta \alpha_{k l}+\Delta \epsilon_{k l}-\Delta \epsilon_{k l}^{*}\right)
$$

Eshelby (1957) has shown that the stress and strain fields inside the inhomogeneity are uniform, and are determined by the expression

$$
\Delta \epsilon_{i j}=S_{i j k l}\left(\theta \Delta \alpha_{k l}+\Delta \epsilon_{k l}^{*}\right)
$$

where

$$
S_{i j k l}=\frac{5 \nu-1}{15(1-\nu)} \delta_{i j} \delta_{k l}+\frac{4-5 \nu}{15(1-\nu)}\left(\delta_{i k} \delta_{j l}+\delta_{i l} \delta_{j k}\right)
$$

for a spherical inclusion in an isotropic matrix, and $\Delta \alpha_{i j}=\alpha_{i j}^{*}-\alpha_{i j}$. Solving (A1), (A2), and (A3) for $\Delta \epsilon_{i j}^{*}$ and inserting the result into (A1) we find that the residual stress in the grain is given by

$$
\Delta \boldsymbol{\sigma}=-\theta \mathbf{C}(\mathbf{I}-\mathbf{S})\left[\mathbf{I}+(\mathbf{C}+\Delta \mathbf{C S})^{-1} \Delta \mathbf{C}(\mathbf{I}+\mathbf{S})\right] \Delta \alpha
$$

In (A5), $\Delta C_{i j k l}=C_{i j k l}^{*}-C_{i j k l}$, and I denotes the fourth-order identity tensor. Evidently, the term in $\Delta \mathbf{C}$ in (A5) represents the effect of the elastic anisotropy of the grains. When $\Delta \mathbf{C}=\mathbf{0}$ 
i.e., when the solid is thermally anisotropic but elastically isotropic, then (A5) reduces to

$$
\Delta \sigma=-\theta \mathbf{C}(\mathbf{I}-\mathbf{S}) \Delta \boldsymbol{\alpha}
$$

The general expression (A5) can be recast in this form by introducing an effective thermal expansion tensor of the grain defined as

$$
\Delta \alpha^{\text {eff }}=\left[\mathbf{I}+(\mathbf{C}+\Delta \mathbf{C S})^{-1} \Delta \mathbf{C}(\mathbf{I}-\mathbf{S})\right] \Delta \alpha
$$

This effective thermal expansion tensor accounts for both the thermal and elastic anisotropy of the grains. The results of Ortiz and Molinari (1988) can be extended to the case of elastic anisotropy by replacing the thermal expansion coefficients of the grain in (2.2) by the effective values defined by (A7).

This result may be used to estimate the effect of elastic anisotropy on the residual stresses in alumina, which has an hexagonal closed-packed structure with elastic moduli: $c_{11}=465$ $\mathrm{GPa}, c_{33}=563 \mathrm{GPa}, c_{44}=233 \mathrm{GPa}, c_{66}=124 \mathrm{GPa}, c_{12}=117$ $\mathrm{GPa}, c_{13}=101 \mathrm{GPa}$, and thermal expansion coefficients: $\alpha_{1}=\alpha_{2}=8.3 \times 10^{-6}{ }^{\circ} \mathrm{C}^{-1}, \alpha_{3}=9.0 \times 10^{-6}{ }^{\circ} \mathrm{C}^{-1}$. Take as effective elastic moduli $E=425 \mathrm{GPa}, \nu=0.21$, and as effective thermal expansion coefficient $\alpha=8.8 \times 10^{-6}{ }^{\circ} \mathrm{C}^{-1}$. Then (A7) gives: $\alpha_{1}^{\text {eff }}=\alpha_{2}^{\text {eff }}=8.297 \times 10^{-6}{ }^{\circ} \mathrm{C}^{-1}, \alpha_{3}^{\text {eff }}=9.043 \times 10^{-6}{ }^{\circ} \mathrm{C}^{-1}$, which differ negligibly from the actual thermal expansion coefficients. Therefore, if follows from this estimate that the effect of the elastic anisotropy of the grains on the average residual stresses is negligible in the case of alumina. However, this does not necessarily imply that elastic anisotropy has no effect on microcracking. The anisotropy may still significantly affect local variations of stress near grain boundaries and triple grain junctions (Tvergaard and Hutchinson, 1987).

\section{References}

Blendell, J. E., and Coble, R. L., 1982, "Measurement of Stress Due to Thermal Expansion Anisotropy in $\mathrm{Al}_{2} \mathrm{O}_{3}$," J. Am. Ceram. Soc., Vol. 65, No. 3, pp. 174-178.

Bao, G., and Suo, Z., "Remarks on Crack-Bridging Concepts," submitted for publication.

Boas, W., and Honeycombe, R. W. K., 1946, "The Plastic Deformation of Non-Cubic Metals by Heating and Cooling," Proc. Roy. Soc. Londong, Series A186, pp. 57-71.

Bower, A. F., and Ortiz, M., 1990, "Solution of Three-Dimensional Crack Problems by a Finite Perturbation Method," J. Mech. Phys. Solids, Vol. 38, No: 4 , pp. 443-480.

Bower, A. F., and Ortiz, M., 1991, "A Three-Dimensional Analysis of Crack Trapping and Bridging by Tough Particles," J. Mech. Phys. Solids, Vol. 39, No. 6, pp. 815-858.

Bower, A. F., and Ortiz, M., 1993, "An Analysis of Crack Trapping by Residual Stresses in Brittle Solids," to appear in ASME Journal of Applied Mechanics.

Budiansky, B., and O'Connell, R. J., 1976, "Elastic Moduli of Cracked

Solid," Int. J. Solids Structure, Vol. 12, pp. 81-97.

Budiansky, B., 1986, "Micromechanics II," Proc. 10th Natl. Congress of Applied Mechanics, P. J. Lamb, ed., ASME, pp. 25-32.

Clarke, F. J. P., 1964, "Residual Strain and the Fracture Stress-Grain Size Relationship in Brittle Solids," Acta Met., Vol. 12, pp. 139-143.

Cleveland, J. J., and Bradt, R. C., 1978, "Grain Size/Microcracking Relations for Pseudo-Brookite Oxides," J. Am. Ceram. Soc., Vol. 61, No. 11-12, pp. $478-481$

Cook, R. F., Lawn, B. R., and Fairbanks, C. J., 1985, "MicrostructureStrength Properties in Ceramics," J. Amer. Ceram. Soc., Vol. 68, pp. 604-615 and 616-623.

Davidge, R. W., and Green, T. J., 1968, "Strength of Two-Phase Ceramic/ Glass Material," J. Mater. Sci., Vol. 3, pp. 629-634.

Evans, A. G., 1978, "Microfracture from Thermal Expansion AnisotropyI. Single Phase Systems," Acta Metall., Vol. 26, pp. 1845-1853.

Evans, A. G,, 1984, “Aspects of Reliability of Ceramics," Defect Properties and Processing of High-Tech Nonmetallic Materials, J. H. Crawford, Y. Chen, and W. A. Sibley, eds., North Holland, pp. 63-80.
Evans, A. G., and Faber, K. T., 1981, "Toughening of Ceramies by Circumferential Microcracking," J. Am. Ceram. Soc., Vol. 64, No. 7, pp. 394-398.

Evans, A. G., and Fu, Y,, 1985, "Some Effects of Microcracks on the Mechanical Properties of Brittle Solids II: Microcrack Toughening," Acta Met. Vol. 33, pp. 1525-1531

Faber, K. T., and Evans, A. G., 1983, "Crack Deflection Processes I: The ory," Acta Met., Vol. 31, No. 4, pp. 565-576.

Faber, K. T., and Evans, A. G., 1983, "Crack Deflection Processes II: Experiment," Acta Met., Vol. 31, No. 4, pp. 577-584.

Fredrich, T. J., and Wong, T. F., 1986, "Micromechanics of Thermally In duced Cracking in Three Crustal Rocks," J. Geophys., Res. B, Vol. 91, No. 12, pp.743-764.

Fu, Y., and Evans, A. G., 1985, "Some Effects of Microcracks on the Mechanical Properties of Brittle Solids," Acta Metall., Vol. 33, pp. 1515-1523.

Gao, H., and Rice, J. R., 1986, "Shear Stress Intensity Factors for a Planar Crack with Slightly Curved Front," ASME Journal of Applied Mechanics, Vol. 53, pp. 774-778.

Gao, H., and Rice, J. R., 1987, "Somewhat Circular Tensile Cracks," Int.

J. Fracture, Vol. 33, pp. 115-174.

Hutchinson, J. W., 1987, "Crack Tip Shielding by Microcracking in Brittle Solids," Acta Met., Vol. 35, No. 7, pp. 1605-1619.

Krstic, V. D., 1984, "Fracture of Brittle Solids in the Presence of Thermoelastic Stresses," J. Am. Ceram. Soc., Vol. 67, pp. 589-593.

Kuszyk, J. A., and Bradt, R. C., 1973, "Influence of Grain Size on Effects of Thermal Expansion Anisotropy in $\mathrm{MgTi}_{2} \mathrm{O}_{5}, " J$. Am. Ceram. Soc., Vol. 56, No. 8 , pp. $420-423$.

Lange, F. F., 1970, "The Interaction of a Crack Front with a Second Phase Dispersion,", Phil. Mag., Vol. 22, pp. 983-992.

Laures, J. P., and Kachanov, M., 1991, "Three-Dimensional Interactions of a Crack Front with Arrays of Penny-Shaped Microcracks,"' Int. J. Fract., Vol 48, pp. 255-279.

Laws, H., and Lee, J. C., 1989, "Microcracking in Polycrystalline Ceramics: Elastic Isotropy and Thermal Anisotropy," J. Mech. Phys. Solids, Vol. 37, No 5, pp. 603-618.

Ortiz, M., 1987, "A Continuum Theory of Crack Shielding in Ceramics," ASME Journal of Applied Mechanics, Vol. 54, pp. 54-58.

Ortiz, M., 1988, "Microcrack Coalescence and Macroscopic Crack Growth Initiation in Brittle Solids," Int. J. Solids Struct., Vol. 24, No. 3, pp. 231-250. Ortiz, M., and Molinari, A., 1988, "Microstructural Residual Stresses in Ceramic Materials," J. Mech. Phys. Solids, Vol. 36, No. 4, pp. 385-400.

Ortiz, M., and Suresh, S., 1993, "Statistical Properties of Residual Stresses and Intergranular Fracture in Ceramic Materials," ASME Journal of Applied Mechanics, in press.

Pohanka, R. C., Rice, R. W., and Walker, B. E., Jr., 1976, "Effect of Internal Stress on the Strength of $\mathrm{BaTiO}_{3}$," J. Am. Ceram. Soc., Vol. 59, pp. 71-74.

Rice, J. R., 1985, "First Order Variation in Elastic Fields Due to Variation in Location of a Planar Crack Front," ASME Journal of Applied Mechanics, Vol. 52, pp. 571-579.

Rice, J. R., 1989, "Weight Function Theory for Three-Dimensional Elastic Crack Analysis," Fracture Mechanics: Perspectives and Directions (Twentieth Symposium), ASTM-STP-1020, R. P. Wei and R. P. Gangloff, eds., American Society for Testing and Materials, Philadelphia, pp. 29-57.

Rice, R. W., and Pohanka, R. C., 1979, "The Grain Size Dependence of Spontaneous Cracking in Ceramics," J.Am. Ceram. Soc., Vol. 62, pp. 559563.

Rice, R. W., Freiman, S. W., and Becher, P. F., 1981, "Grain-Size Dependence of Fracture Energy in Ceramics: I. Experiment," J. Am. Ceram. Soc., Vol. 64, pp, 345-350.

Rose, L. R. F., 1975, "Toughening Due to Crack Front Interaction with a Second Phase Dispersion,"' Mechanics of Materials, Vol. 6, pp. 11-15.

Siebeneck, H. J., Hasselman, D. P. H., Cleveland, J. J., and Bradt, R. C., 1976, "Effect of Microcracking on the Thermal Diffusivity of $\mathrm{Fe}_{2} \mathrm{TiO}_{5}$," J. Am. Ceram. Soc., Vol. 59, pp. 241-244.

Singh, J. P., Virkar, A. V., Shetty, D. K., and Gordon, R. S., 1979, "StrengthGrain Size Relations in Polycrystalline Ceramics," J. Am. Ceram. Soc., Vol. 62 , pp. $179-183$

Suresh, S., 1985, "Fatigue Crack Deflection and Fracture Surface Contact: Micromechanical Models," Metall. Trans., Vol. 16A, pp. 249-260.

Swain, M. V., 1986, " $R$-Curve Behavior in Polycrystalline Alumina Material," J. Mater. Sci. Lett., Vol. 5, pp. 1313-1315.

Swanson, P. L. Fairbanks, C. J., Lawn, B. R., Mai, Y. W., and Hockey, B. J., 1987, "Crack Interface Grain Bridging as a Fracture Resistance Mechanism in Ceramics I: Experimental Study on Alumina," J. Am. Ceram. Soc., Vol. 70, No. 4, pp. 279-289.

Tvergaard, V., and Hutchinson, J. W., 1988, "Micro-Cracking in Ceramics Induced by Thermal Expansion or Elastic Anisotropy," J. Am. Ceram. Soc., Vol. 71, p. 157

Vekinis, G., Beaumont, P. W. R., and Ashby, M. F., 1990, " $R$-curve Behavior of $\mathrm{Al}_{2} \mathrm{O}_{3}$ Ceramics," Acta Met., Vol. 38, No. 6, pp. 1151-1162. 